\title{
Chapter 12 \\ Integrating Gender to Improve HIV Services in Uganda
}

\author{
Taroub Harb Faramand
}

\begin{abstract}
The Partnership for HIV-Free Survival (PHFS) was a regional initiative supported by the World Health Organization (WHO), the US President's Emergency Plan for AIDS Relief (PEPFAR), the US Centers for Disease Control and Prevention (CDC), and the U.S. Agency for International Development (USAID) that applied a quality improvement (QI) approach in a select number of demonstration sites in six countries to improve the prevention of mother-to-child transmission of HIV and increase HIV-free survival of exposed infants. The Ivukula Health Center was one of the chosen demonstration sites in Uganda, one of the six PHFS focus countries. This case study describes how the Ivukula Health Center QI team improved the quality of HIV services and applied the same QI methods to integrate gender considerations into the process improvements they tested. The case illustrates how engaging male partners and community members in improving care can play a critical role in improving health outcomes and discusses a generic approach to integrating gender considerations into any development activity.
\end{abstract}

Keywords Gender integration - Prevention of mother-to-child transmission of $\mathrm{HIV} \cdot$ Retention in care $\cdot$ Uganda

\section{Background}

Gender is a critical component of improving healthcare quality because to improve healthcare quality for all, we have to identify and address gaps in quality between women and men and girls and boys. Data and experience show that clients, family and friends, communities, and health providers are all influenced by the culture they live in and by that culture's perspective on gender. Thus, a gender-sensitive approach

\footnotetext{
T. H. Faramand $(\triangle)$

WI-HER, LLC, Vienna, VA, USA

e-mail: tfaramand@wi-her.org
} 
takes into account the different needs, constraints, and opportunities of women, men, girls, and boys and responds to them strategically in program design, implementation, and evaluation. Box 12.1 describes the generic steps in integrating gender considerations into any development activity.

Ivukula is a rural community located in Uganda's Namutumba District, with six sub-counties and a population of 254,000 people. Agriculture is the main economic activity in Ivukula, and $80 \%$ of farmers practice subsistence agriculture. The health center in Ivukula serves a catchment population of 45,000.

In early 2013, the Partnership for HIV-Free Survival (PHFS), a regional initiative supported by the World Health Organization (WHO), the US President's Emergency Plan for AIDS Relief (PEPFAR), the US Centers for Disease Control and Prevention (CDC), and the U.S. Agency for International Development (USAID), initiated work to prevent mother-to-child transmission of HIV and increase HIV-free survival. PHFS used a quality improvement (QI) approach in a select number of demonstration sites in each country to learn how to better organize care delivery; in Uganda, the Ivukula Health Center was one of the chosen demonstration sites.

\section{Box 12.1 Steps in Gender Integration}

Gender integration refers to strategies applied in activity assessment, design, implementation, and evaluation to take gender norms into account and to compensate for gender-based inequalities. Improvement approaches provide an effective framework to identify and address the different needs, constraints, and opportunities of men, women, girls, and boys, improving outcomes for all and closing gender-related gaps.

The six-step process listed below outlines how improvement teams can integrate gender in quality improvement activities and implementation:

- Step 1: Conduct a gender analysis to inform program design and implementation.

- Step 2: Collect and analyze sex-disaggregated and gender-sensitive data.

- Step 3: Identify gender-related gaps and issues and develop changes to test.

- Step 4: Implement and monitor gender-related changes over time to determine whether desired results are achieved.

- Step 5: Scale up effective changes to close gender-related gaps.

- Step 6: Document and share learning.

For more explanation, see A Guide to Integrating Gender in Improvement, available at https://pdf.usaid.gov/pdf_docs/PA00TC12.pdf 


\section{Organizing the Improvement Effort}

\section{Beginning the PHFS Pilot Effort}

Uganda's Ministry of Health (MOH), in partnership with USAID-funded partners, launched PHFS in April 2013 at 22 facilities, including Ivukula, in six districts in Uganda. PHFS aimed to address its goal of preventing mother-to-child transmission of HIV and increasing HIV-free survival by ensuring HIV-positive mothers were identified and retained in care and treatment, promoting optimal infant feeding practices (in accordance with WHO Guidelines on HIV and Infant Feeding), and supporting HIV-positive mothers' overall health and nutrition - in effect, integrating HIV, well-baby, and nutrition services for mother-infant pairs.

The national team leading the PHFS initiative in Uganda identified, as an integral component of the project's primary goal, developing providers' capacity to identify and respond to gender-related gaps in services or programs.

\section{Obtaining Local Buy-in}

A USAID-funded project, with decades of experience in applying quality improvement approaches to improve health outcomes, was the implementing partner for PHFS in Uganda. For this work, the USAID-funded project planned a strategy that would include gender considerations as part of the pilot effort. To support gender integration, they brought on board technical advisors with significant experience and expertise in addressing gender concerns in health using quality improvement approaches.

As a first step, the USAID-funded project's senior gender advisor sought "buyin" from the Namutumba district health officer. The senior gender advisor was aware that to ensure the long-term success of the project, this step had to be more than routine and perfunctory. She had to begin by sparking sincere enthusiasm among key players, officials, or other stakeholders, who were able to influence and impact others.

The senior gender advisor began her meeting with the Namutumba district officer by sharing how addressing gender can improve health outcomes. In an effort to directly address topics often avoided because of cultural norms, she raised subjects that might resonate with the district officer's cultural and professional experience. In particular, the senior gender advisor pointed out that men were often concerned that they would be made to feel guilty and responsible for poor health outcomes. She talked about how, instead, awareness about gender concerns might improve interpersonal communication and increase women and men's compliance with their healthcare regimens. During the meeting, the senior gender adviser encouraged the district officer to share his own concerns about implementing gender considerations into the QI process. 
Honest and open conversation yielded results: When the district officer was given an opportunity to express his own vision for integrating gender to improve the district's health services, he grew enthusiastic about the possibility of significant results.

\section{Formation of the QI Team}

Based on the district officer's familiarity with and knowledge of the district's health centers, he and the senior gender officer selected the Ivukula facility to pilot the gender interventions.

In February 2013, the district officer recruited a district coach based on leadership skills, motivation, and interest. The district coach's role included working with the project's QI advisor to assemble clinic teams and launch the QI effort, conduct training sessions for clinic staff, provide coaching support, and offer guidance and mentoring to QI team members. In February 2013, the project's QI advisor conducted a QI training for the Namutumba district coach along with coaches selected from several other pilot districts.

Improvement work in the Ivukula facility started in February 2013, 2 months prior to the official launch of PHFS at all pilot sites. Before visiting the Ivukula facility to recruit a QI team, the USAID-funded project's QI advisor and the district coach planned how they would address possible reservations and concerns from the clinic staff. During the February visit, they explained QI principles to the clinic staff and listened as staff members cited an already heavy workload and expressed reservations about gender concerns and integrating a gender component in QI interventions. The district coach and the project's QI advisor were aware that frontline healthcare workers were likely to have concerns about trying to address longstanding gender issues and social norms-issues that they saw as intractable and beyond their responsibility as clinicians. They addressed the staff's concerns by helping them envision the potential results of putting gender-sensitive QI interventions into place.

Gradually, as concerns were aired, enthusiasm for the initiative began to grow and the staff began to recruit a team. The coach and QI advisor made it clear to the clinic staff that participation was entirely voluntary and those nominated could refuse their nomination. However, they found that the staff were excited and eager to participate.

The clinic staff chose a nine-member QI team from diverse parts of the clinic, including the maternity ward, laboratory, and outpatient clinic. As their leader, team members selected a clinical officer who was widely respected for her leadership skills and commitment to her clients. In addition, the clinic staff recommended that a male expert client and a mentor mother also join the QI team, to support with outreach and ensure that community and patient perspectives were represented on the team. 
The expert client was a male HIV-positive patient successfully managing his care. He encouraged men to test, enroll in care, and support their female partners and babies who were in care. The mentor mother was an HIV-positive mother who had successfully prevented transmission of HIV to her baby by enrolling and keeping herself and her baby in care. Her role was to share her personal experience and encourage women to adhere to appointments and their HIV treatment regimen. In addition, the mentor mother encouraged women to involve their male partners in care. As initial incentives to participate on the improvement team, project staff provided the mentor mother and expert client with training on health education.

\section{Training Heightens Staff Awareness of Gender Norms}

The district QI coach and project QI advisor provided training on incorporating gender in the improvement approach for the Ivukula team in March 2014. Because the facility team was being asked to pioneer gender interventions, a substantial part of the training was devoted to helping clinic staff identify and address genderrelated gaps that prevented attaining the desired outcomes. The training presented a version of the model for improvement with plan-do-study-act cycles adopted to focus on gender-specific QI interventions (Fig. 12.1).

Staff were trained to adhere to the "do no harm" principle to avoid causing harm to their patients. In quality improvement, the "do no harm" principle means that the changes implemented should never intentionally or unintentionally harm participants. To do this, it is vital to consider how a change idea will affect different groups of people_-and whether it might harm one group. For example, staff were trained to always ask HIV-positive women if they wanted to invite their male partners to the

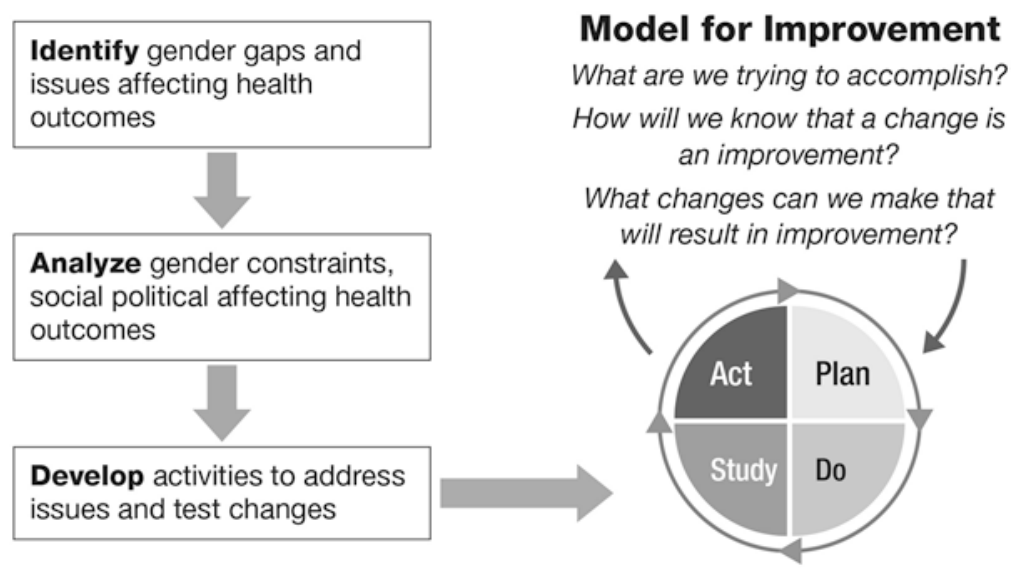

Fig. 12.1 Integrating gender within the model for improvement and plan-do-study-act cycles 
facility because some women can be put at risk of partner violence if the clinic invites their male partners or discloses their HIV status against a patient's wishes.

\section{Carrying Out the Improvement Effort}

\section{Problem Analysis}

The Ivukula team initially focused on the quality of existing data on retention of mothers and their babies in care. The clinic QI team began an analysis of the services received by mothers and their infants by reviewing and analyzing the clinic's existing medical documents, which consisted primarily of "mother and baby cards" where staff recorded medical and appointment history.

The review revealed that babies and mothers were being seen separately by different providers, and their care was not linked. A large percentage of HIV-exposed infants had not been seen at the facility and were not being provided the care they should have been.

The team found logistical, operational, and administrative performance gaps that they wanted to address. One of the gaps, perhaps the most obvious, was a lack of care coordination due to the mother and baby cards being stored in separate rooms. Another issue was that appointments for mothers and babies were scheduled on different days, making compliance and follow-up more complicated for clients. The third was an issue of data quality: mother-baby cards were not being properly filled out, resulting in incomplete records and making it challenging to tell who accessed services.

The QI team set a primary goal of reaching, by January 2014, a 100\% level in retention in care for mothers and babies. With support from the district QI coach, they decided to change the process of care by offering services to mothers and their babies at the same service point. To do so they developed a new process and patient flow chart.

Efforts to improve retention started with ensuring that mother-baby pairs were supported to attend mother-baby pair appointments. The QI team in Ivukula, in August 2013, directed facility staff to begin pairing mother and baby cards, storing together the health information of mothers and babies.

At the same time, the QI team also directed staff to ensure the next appointments for both mothers and babies were made for the same day. Appointments were given at the end of the visit by one person at the facility who scheduled an initial appointment for the mother and a follow-up appointment for baby and mother. The QI team reported reduced waiting time for mothers, reduced repeated visits, and streamlined services, which resulted in reducing staff workload.

According to the QI team leader, "At the beginning we felt overwhelmed and we were worried about increased load of work, but when we saw improvements, we were so excited and became motivated to continue." 


\section{Support for the QI Team}

The USAID-funded project's Uganda staff, through monthly visits to Ivukula, supported the QI team to identify gaps in care, prioritize areas for improvement, and develop and test change ideas to address the gaps and implement these changes.

The senior gender advisor provided ongoing in-person and virtual support to project staff, including building their capacity to lead a coaching session related to gender and integrate gender into QI for staff at the health facility. In April 2013, she trained the USAID-funded project's Uganda staff on gender integration. Training included sensitizing the team to gender norms and issues, including providing general information about how gender affects people's daily lives, as well as specific information about how to integrate gender in improvement activities. This training enabled the USAID technical assistance project staff in Uganda to incorporate gender considerations affecting health outcomes as an integral component of the QI process.

The senior gender advisor supported the Ivukula QI team to document gender integration activities through development of simple tools for the QI team to use to integrate gender, including a driver diagram, which identified the contributing factors to lack of male participation in PMTCT services and to women's inability to keep appointments and remain in care due to child care responsibilities or financial constraints in addition to disclosure issues. She suggested possible activities to address gender-related drivers affecting outcomes and developed scenarios for the clinic team to use to discuss and better understand how gender gaps affected achieving their goals and improving outcomes. The senior gender advisor also provided information about best practices and activities that worked well to close gender gaps, including involving men. Table 12.1 outlines the timeline of gender support to the Ivukula QI team.

Table 12.1 Timeline of gender technical support to Ivukula Health Center

\begin{tabular}{l|l}
\hline April 2013 & $\begin{array}{l}\text { Senior gender advisor provided an interactive training for and technical } \\
\text { support to the Uganda project team on methods and illustrative action steps to } \\
\text { integrate gender in all phases of improvement work }\end{array}$ \\
\hline August 2013 & $\begin{array}{l}\text { Senior gender advisor provided a follow-up gender training for staff and } \\
\text { technical support to adopt learning session materials to address gender issues } \\
\text { and develop training materials to use for gender trainings }\end{array}$ \\
\hline March 2014 & $\begin{array}{l}\text { Project staff provided a training on gender integration to Ivukula staff and met } \\
\text { with the district health office to discuss gender integration approaches to } \\
\text { improve outcomes }\end{array}$ \\
\hline July 2014 & $\begin{array}{l}\text { Senior gender advisor supported project staff in supporting QI teams to } \\
\text { integrate gender in QI activities, as well as provided direct support in gender } \\
\text { integration to QI teams }\end{array}$ \\
\hline September & $\begin{array}{l}\text { Senior gender advisor supported Ivukula to document gender integration } \\
\text { activities }\end{array}$ \\
\hline $\begin{array}{l}\text { October } \\
2015-\text { August }\end{array}$ & $\begin{array}{l}\text { Senior gender advisor provided ongoing virtual support to project staff, } \\
\text { including building their capacity to lead a coaching session related to gender } \\
\text { considerations and to integrate gender into QI for staff at the health facility }\end{array}$ \\
\hline
\end{tabular}




\section{Gender Interventions}

Shortly after starting improvement work, midwives and other providers started to realize how much and how often their women clients talked about the role their partners and families played in making decisions about health-related matters. QI team members noted that women clients had long been raising these issues; however, without a way to address them, clients' questions and concerns went unanswered. The gender training and efforts to integrate gender considerations in QI efforts equipped service providers with a tool to begin to address the gender concerns.

During counseling sessions and in less formal conversations, women were revealing to midwives and other providers, as well as to mentor mothers and expert clients, how often and how much gender-related concerns impacted care. Mentor mothers and expert patients both reported, through informal interviews, that mothers who had not disclosed their HIV status to their partners often failed to access services because they did not want to be questioned about their whereabouts. Other mothers reported competing priorities in the household and a lack transport or other forms of family support to enable them access services in the health facility.

The clients expressed that they often feared the possibility of physical violence if they disclosed their HIV-positive status to their partners. Mothers also felt that their partners were not likely to understand the importance of HIV services and remaining in care.

After the QI team began to recognize how heavily gender concerns affected women's decisions about whether to remain in care, members decided to test whether increasing male partner involvement might mitigate the identified reasons women were not being retained in care. Change interventions to increase male partner involvement were put in place. Changes included: (1) joining with other PHFS implementing partners to reach out in the community to encourage men to accompany their female partners to the clinic regardless of the male partners' HIV status, (2) involving community leaders in encouraging men to accompany their wives or partners, (3) counseling women about the benefits of involving male partners to accompany them on clinic visits, (4) inviting male partners to attend clinic visits (per the client's wishes), and (5) offering health services to male partners at the clinic, such as measuring blood pressure and weight.

With support from the senior gender advisor, the Ivukula QI team was able to respond specifically to these gender-related issues. Male partners, the team discerned, might be motivated to attend clinic appointments because of their love and concern for their partners and children. In addition, health education and services might serve as incentives for men to come to the clinic, so efforts to involve male partners should consider such incentives. At the same time, health workers needed to be aware of an underlying concern in which men felt that efforts to address gender concerns meant that blame would be directed toward males for genderrelated issues. 
In August 2013, the improvement team at the Ivukula facility began an effort to raise awareness among clients and their partners, as well as in the community, about the need to involve men. They first talked about how to shape the intervention; i.e., whether men coming to the clinic was the only way that they could be considered involved. The team discussed whether male involvement could be defined as men being regularly tested for HIV or providing their female partners with nutritional, psychological, and financial support. Finally, for purposes of this initial intervention, the team decided to measure results by assessing the number of men who accompanied their female partners and babies to the facility.

The clinic improvement team encouraged community leaders to invite men to come to the facility. In addition, they initiated an effort to reach out to men by joining in ongoing community outreach efforts being conducted by two other PHFS implementing partners. The implementing partners set about raising awareness to encourage men to accompany their female partners to the clinic. Outreach representatives were usually able to talk to men about this when they came to the market. Sometimes, they were able to engage the men at religious events.

In February 2014, the clinic QI team identified a prime opportunity to further engage men in participating with their wives and children. The clinic had been offering monthly family support group meetings in which men and women came for health education and to share their own HIV experiences with others. They brought their babies below 2 years of age. During those meetings, no drug distribution took place; HIV-positive men and women had to return during the same month to receive their medications. The QI team noticed that women who attended the family support group meetings did not always return for their follow-up visits; their husbands or male partners would come to the clinic to pick up antiretroviral medications for both themselves and their wives or partners. HIV-negative men did not join these meetings as they felt they did not concern them. The improvement team identified an opportunity to utilize the meetings to engage men and keep mothers and their babies in care.

The improvement team put in place a change to synchronize family support group meetings and clinic appointments for mother-baby pairs and males. Beginning in March 2014, the Ivukula facility began to focus health talks on male partners' role in preventing mother-to-child transmission of HIV (PMTCT). The site team also offered couple's counseling during special visits when the first tests of infants were done to assess the infant's HIV status.

"Male partners are eager to know the HIV results of their exposed infants and they are motivated to support their partners when they understand their roles and responsibilities...we ensure that we always give them health talks in relation to available services for both the mother and male partner to protect the exposed infants from risk of contracting HIV infection," the team reported. Furthermore, the team leader reported that, "Men became so engaged in their babies' health that if the nurse or the midwife would miss performing any of the follow-up items, fathers would ask why... They demand a good service."

Clinic staff expanded the family support group meetings to invite serodiscordant couples-where one partner is HIV negative and the other is HIV positive. 
Couples became so interested in the health education sessions that they took active participation in selecting session topics. The team broadened the range of health services offered to male partners to encourage them to participate in counseling sessions. Previously, they had received services at the outpatient clinic only when they were sick or came for their medication. Clinic staff began offering blood pressure and weight measurements as well as HIV counseling and testing. In addition, the men were offered a nutritional status assessment. These incentives led to more male partners coming to the facility with their female partner. The staff shared that it contributed to driving up retention rates of mother-baby pairs in care (Fig. 12.2).

The mentor mother and expert client played key roles in implementing genderrelated changes. Both had received training in gender integration and were sensitized to the gender issues and gaps that men and women might be affected by or face. Being sensitized to gender issues means they became aware that service providers might treat male or female clients differently when delivering health services and that gender issues may affect client willingness to seek services, continue to use services, and carry out the health behaviors advocated by healthcare providers.

With her knowledge and experience of the culture and mothers' issues, the mentor mother could successfully ensure that the issue of disclosure could be addressed without causing harmful consequences. She supported mothers who expressed a clear desire to disclose their status to their partner. The team members strongly adhered to a "do no harm" approach to make sure that no woman was forced to disclose status. They took a gender-sensitive approach in which members carefully

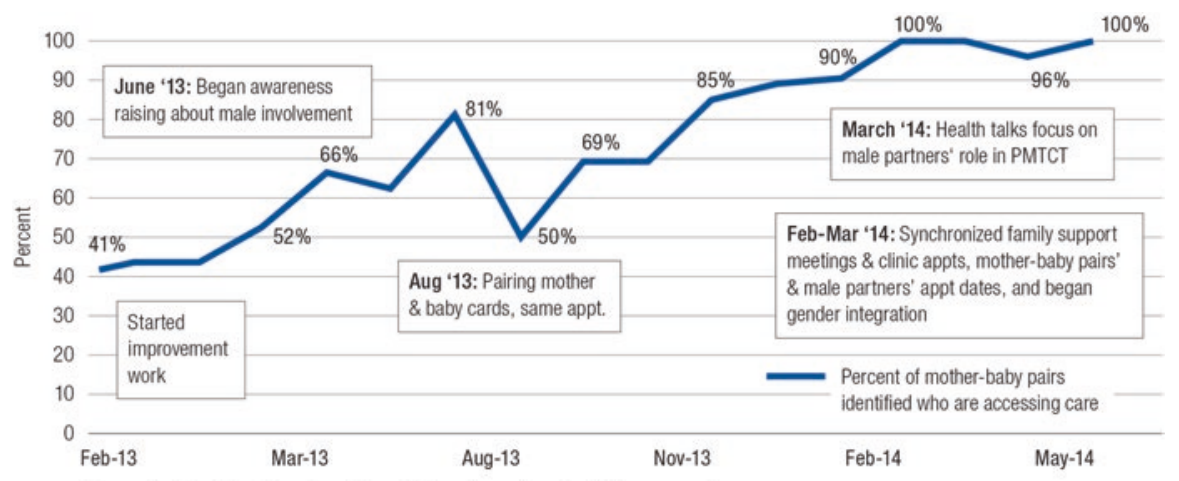

Denominator: Number of mother-baby pairs who should be accessing care

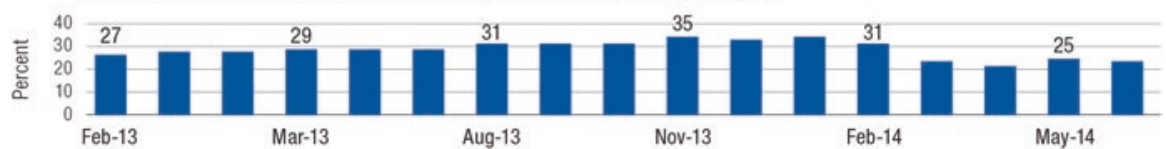

Fig. 12.2 Mother-baby pairs retained in care at Ivukula Health Center, February 2013-June 2014 
made sure that male involvement and disclosure were supported, but optional, and always ultimately the decision of the female client. The team maintained a strong awareness that engaging male partners and other family members, such as mothersin-law, should never lead to negative unintended consequences.

The expert client supported the implementation of a gender-sensitive approach by linking the QI team with the community. He helped make people in the community aware of how important it was for male partners and husbands to support their wives or female partners. The expert client and QI team members were aware that sensitization of community members would require time. The mentor mother and the expert patient visited homes and provided counseling messages to both women and men in the community. The expert client invited community members to attend clinic events. Speakers in health education sessions and clinic staff repeatedly addressed the topic of men participating in their partners' health during health talks, immunization sessions, and nutrition group counseling sessions. Team members, including the expert client, also engaged clients in person-to-person conversations, taking advantage of such times as when clients were waiting for services.

\section{Results}

\section{Ivukula Health Center}

Improvements in outcomes at Ivukula as well as all the pilot facilities came about as the result of both logistical changes, such as pairing mother-baby cards, and genderrelated changes. As of January 2014, the retention rate of mother-baby pairs surpassed $85 \%$ at more than 20 of the pilot clinics, up from $22 \%$ at the baseline in April 2013. At the Ivukula facility, retention rates reached $100 \%$ for mother-baby pairs in March 2014, up from $41 \%$ in February 2013 (see Fig. 12.2). The improvement team kept coaching guides and tracked improvement on a handwritten graph as well as electronically in an Excel spreadsheet.

\section{All Pilot Sites}

By February 2014, the 22 pilot sites had all achieved strong gains in retaining mother-baby pairs in care, from $2.2 \%$ of pairs retained in care to over $60 \%$, as seen in Fig. 12.3. The indicator used to track the retention of mother-baby pairs is the number of mother-baby pairs in postnatal care (a count of how many babies came each month and checked with whether their mothers also came in the same month) over the number of mother-baby pairs expected to be in postnatal care (calculated with the number of babies who were ever enrolled in early infant diagnosis, still under 18 months and living, as a proxy for the denominator). 


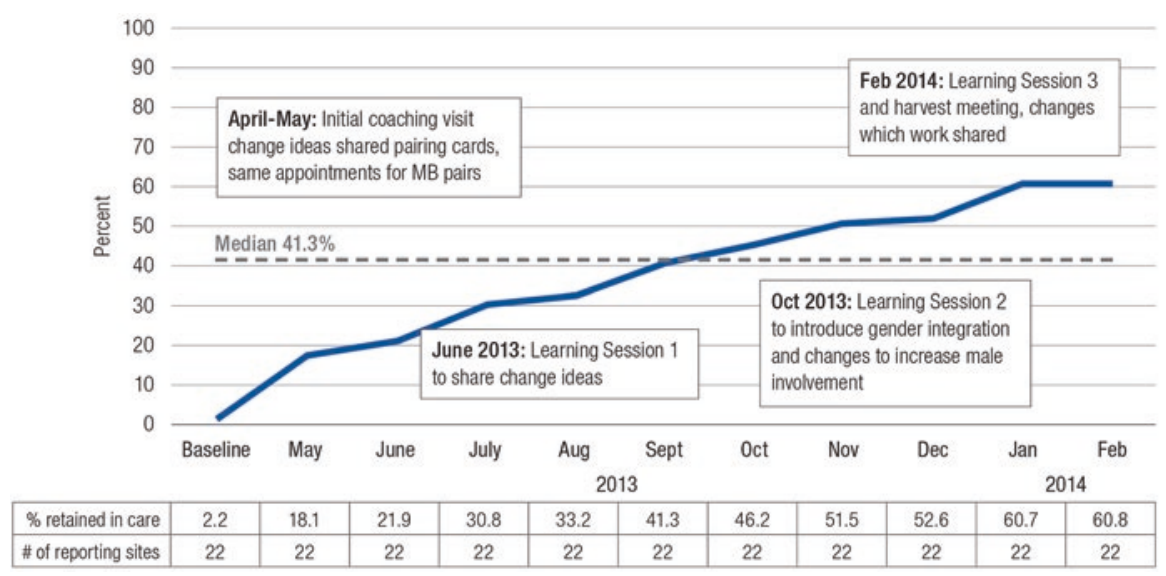

Fig. 12.3 Percentage of mother-baby pairs retained in care at 22 PHFS pilot sites, April 2013February 2014

\section{Team Motivation Grows as They Begin to See Results}

Team members later reported that they were initially skeptical about how effective it would be to review charts and documents they knew to be inadequate. In retrospect, however, they said that were pleasantly surprised to find how much insight the analysis of the cards produced.

Before starting the improvement initiative, while the clinic staff was aware of many of the logistical inefficiencies described above, they were unable to take the time to consider changing the status quo, no matter how unworkable they all knew it to be. In addition, the staff members felt they did not have the authority or time to undertake even a basic task such as putting the cards in the same location.

By implementing a QI process that called for the analysis and identification of gaps and for regular meetings, QI team members were given an opportunity to identify inefficiencies and come up with possible solutions. They were also able to delegate responsibilities to individuals to ensure that solutions were carried out.

\section{Institutionalization}

As the Ivukula facility continued to produce tangible results, the clinic's QI team leader transferred knowledge to scale up gender integration in improvement to neighboring facilities in the Namutumba District (see Box 12.2). In addition, the USAID-funded project team based in Uganda helped to build the capacity of other implementing partners in QI and gender integration. In 2015, Uganda's MOH incorporated the gender component into the MOH's QI training curriculum. 


\section{Box 12.2 Ivukula QI Team's Leader Spreads Knowledge to Other Pilot Sites About Scaling Up Gender Interventions}

In May 2015, the district health officer recognized the leadership potential of the Ivukula QI team leader and made her responsible for bringing knowledge to other clinics. She trained facilities in improvement methodologies and transferred knowledge to scale up gender integration in improvement to neighboring facilities in the Namutumba District. She presented results and challenges and how the team at the Ivukula facility addressed such challenges and achieved their goal of $100 \%$ retention. Further, she visited facilities and worked with the improvement teams to guide them on the improvement process start-up and to share changes that were tested and worked at the Ivukula Health Center. She has since been promoted, now working as an improvement coach at the district level.

\section{Reflection}

This case study illustrates how engaging male partners and community members in improving care can play a critical role in improving health outcomes. The results at Ivukula show how innovative gender integration components can dramatically improve routine care. By analyzing the causes of lack of retention of mother-baby pairs in PHFS-supported services from a gender perspective, the team was able to identify effective methods of increasing retention and improving outcomes for mothers, babies, and male partners.

When men are educated about their partner's status and the health implications of not remaining in treatment, they are more willing to support mothers by facilitating their transportation to the health center and providing nutritional needs for the family. The support of male partners, even if they are not physically able to attend clinic visits with their female partner, is important to retaining mother-baby pairs in care. Key factors that contributed to the success of the work in Ivukula included positive leadership and teamwork and passion among improvement team members.

Using a range of methods and venues to develop community relationships was critical to the team's success. Activities at the Ivukula Health Center show how empowering health workers and providing them with the tools they need can significantly improve the quality of health services. Ivukula shows how building local capacity results in institutionalization and scale-up.

The study also serves as a reminder of how important it is that those working with gender concerns be trained and sensitized to the cultural norms that underlie the issues. Throughout the QI process, technical advisors and the QI team sought to dispel the notion that paying attention to gender concerns meant blaming men for anything that goes wrong.

Ivukula's team built the capacity of healthcare providers to integrate gender issues into efforts to improve care, on the frontlines of health services. By becoming 
aware of social and cultural influences, the team was able to successfully engage male partners in programs focused on eliminating mother-to-child transmission and to educate them about the importance of mothers and babies accessing services, adhering to treatment, and remaining in care. This helped to create champions within families who supported mother-baby pairs to remain in care through the 18-month mark.

Acknowledgments The author expresses special gratitude to Dr. Charles Masinga, District Health Officer in Namutumba, and Ms. Shanifa, Ivukula Quality Improvement Team Leader, for their hard work, professionalism, and commitment to the improvement of healthcare in Uganda and to Ms. Tamara Nsubuga-Nyombi, USAID ASSIST Senior Quality Improvement Advisor in Uganda, and Ms. Joyce Draru, USAID ASSIST Quality Improvement Officer in Uganda, for their leadership in designing and scaling up this improvement effort.

The PHFS initiative described in this case was supported by the USAID Office of HIV/AIDS and the Office of Health Systems through the USAID ASSIST Project, implemented by University Research Co., LLC under Cooperative Agreement Number AID-OAA-A-12-00101.

Open Access This chapter is licensed under the terms of the Creative Commons Attribution 4.0 International License (http://creativecommons.org/licenses/by/4.0/), which permits use, sharing, adaptation, distribution and reproduction in any medium or format, as long as you give appropriate credit to the original author(s) and the source, provide a link to the Creative Commons licence and indicate if changes were made.

The images or other third party material in this chapter are included in the chapter's Creative Commons licence, unless indicated otherwise in a credit line to the material. If material is not included in the chapter's Creative Commons licence and your intended use is not permitted by statutory regulation or exceeds the permitted use, you will need to obtain permission directly from the copyright holder.

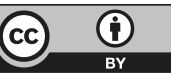

